\title{
Editorial
}

\section{Forefront of Fuzzy Logic in Data Mining: Theory, Algorithms, and Applications}

\author{
Gözde Ulutagay, ${ }^{1}$ Ronald Yager, ${ }^{2}$ Bernard De Baets, ${ }^{3}$ and Tofigh Allahviranloo ${ }^{4}$ \\ ${ }^{1}$ Department of Industrial Engineering, Izmir University, Izmir, Turkey \\ ${ }^{2}$ Iona College, Machine Intelligence Institute, New Rochelle, NY, USA \\ ${ }^{3}$ Department of Mathematical Modelling, Statistics and Bioinformatics, Ghent University, Ghent, Belgium \\ ${ }^{4}$ Department of Mathematics, Islamic Azad University, Tehran, Iran \\ Correspondence should be addressed to Gözde Ulutagay; gozde.ulutagay@izmir.edu.tr
}

Received 8 November 2016; Accepted 8 November 2016

Copyright (C) 2016 Gözde Ulutagay et al. This is an open access article distributed under the Creative Commons Attribution License, which permits unrestricted use, distribution, and reproduction in any medium, provided the original work is properly cited.

Data mining uses various techniques and theories from a wide range of areas for the extraction of knowledge from large volumes of data. However, uncertainty is a widespread phenomenon in data mining problems. The ongoing challenges of uncertainty give rise to a plethora of knowledge extracting methods that use fuzzy logic. The aim of this special issue is twofold:

(i) to present recent outstanding developments and trends in the theory and algorithms of data mining using fuzzy logic,

(ii) to create a multidisciplinary forum for discussion on recent advances in data mining as well as new applications to biology, economics, ecology, engineering, finance, management, medicine, and so forth, using fuzzy logic.

In "Understanding Open Source Software Evolution Using Fuzzy Data Mining Algorithm for Time Series Data" by M. Saini et al., a fuzzy data mining algorithm for time series data is presented in order to generate association rules for evaluating the existing trends and regularity in the evolution of open source software projects.

In "Fuzzy Constrained Probabilistic Inventory Models Depending on Trapezoidal Fuzzy Numbers" by M. F. ElWakeel and K. O. Al-yazidi, two types of the mixture shortage inventory model under varying order cost constraints, with lead time demand under exponential, Laplace, and uniform distributions, are discussed. Also some special cases are handled and comparisons are performed under crisp and fuzzy environments.

In "An Improved Fuzzy Based Missing Value Estimation in DNA Microarray Validated by Gene Ranking" by S. Saha et al., a modified version of the LRFDVImpute technique is proposed to impute multiple missing values of time series gene expression data. The results of imputation by a genetic algorithm (GA) based gene ranking methodology along with some regular statistical validation techniques are presented.

In "Robust FCM Algorithm with Local and Gray Information for Image Segmentation” by H. Barrah et al., a robust variant of the fuzzy c-means clustering algorithm is proposed to eliminate the drawback of its parameter dependency. The proposed algorithm is fully free of the empirical parameters and robust against noise. Moreover, a new factor that includes the local spatial and gray level information is proposed.

In "Fuzzy Aspect Based Opinion Classification System for Mining Tourist Reviews" by M. Afzaal et al., a fuzzy aspect based opinion classification system which efficiently extracts aspects from user opinions is proposed. In order to evaluate the effectiveness of the system, experiments on real world datasets are performed. According to the experimental results of the study, the proposed system is effective in aspect extraction with an improved classification accuracy.

In "Cardinal Basis Piecewise Hermite Interpolation on Fuzzy Data" by H. Vosoughi and S. Abbasbandy, interpolation of fuzzy data by the fuzzy-valued piecewise Hermite polynomial is presented for general case based on the cardinal 
basis functions. Moreover, linear, cubic, and quintic situations are considered for computational examples.

We would like to thank the authors who shared their views and expertise with their excellent works, as well as the reviewers whose objective and critical comments contributed to the quality of our special issue. We strictly hope that the concerning researchers from various fields will find this special issue interesting, thought-provoking, and informative.

Gözde Ulutagay Ronald Yager

Bernard De Baets

Tofigh Allahviranloo 

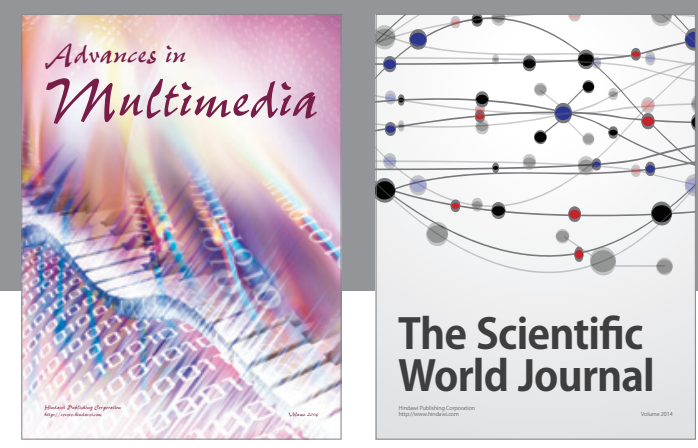

The Scientific World Journal
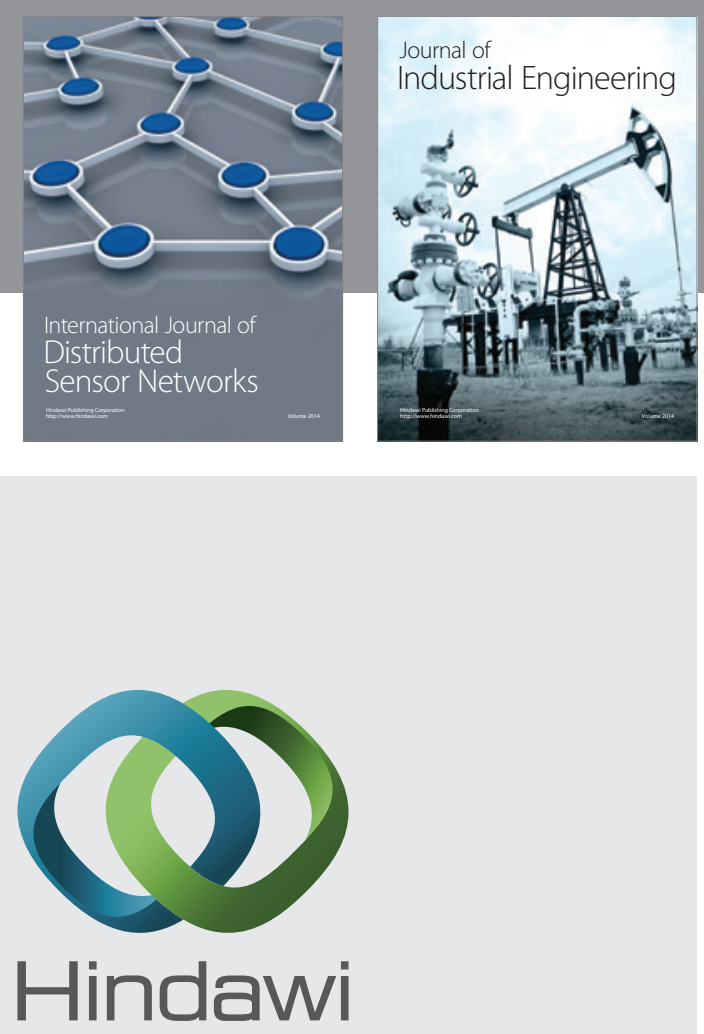

Submit your manuscripts at

http://www.hindawi.com

\section{Computer Networks} and Communications
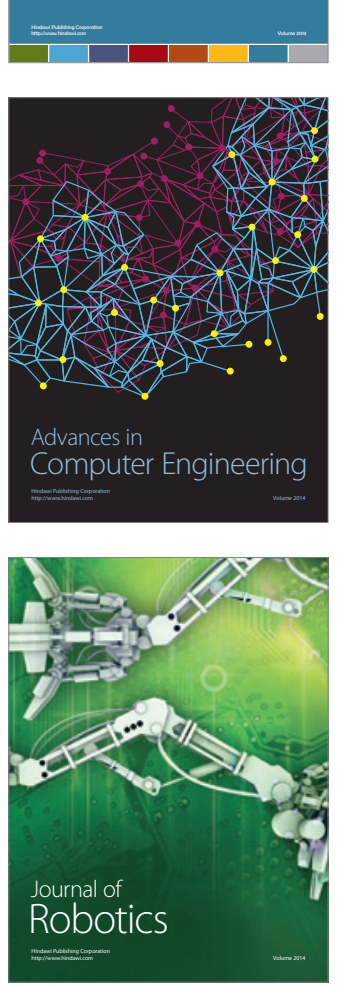
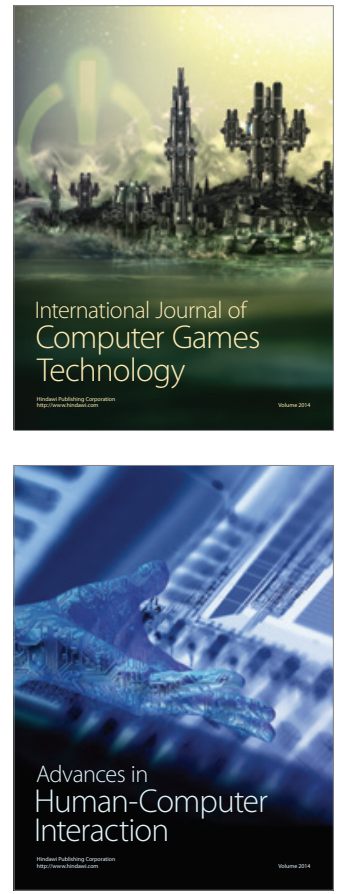
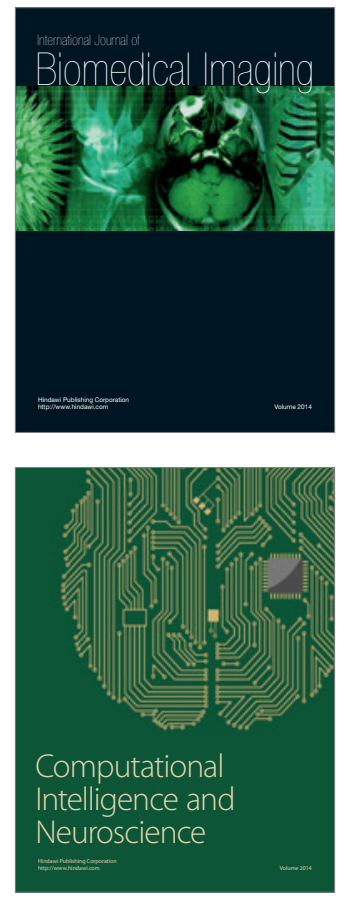
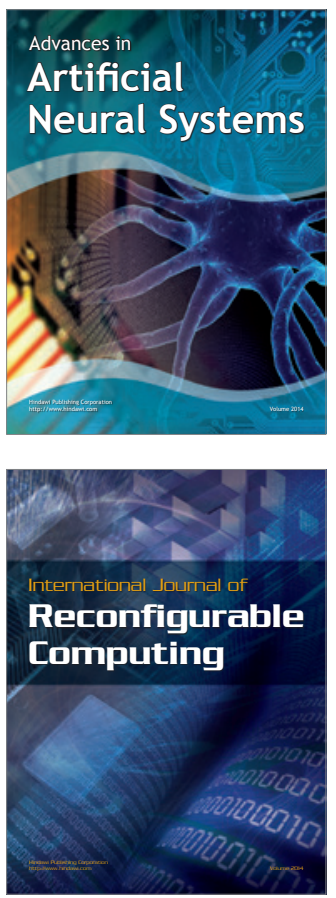
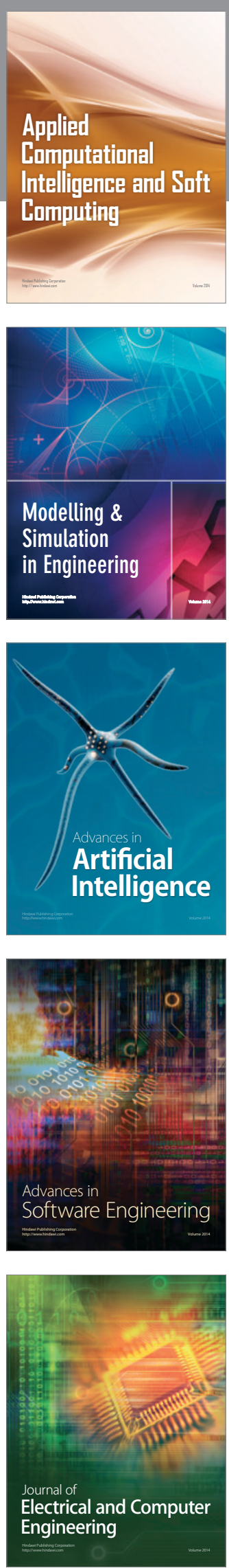